\title{
Use of cyclin-dependent kinase (CDK) 4/6 inhibitors for hormone receptor-positive, human epidermal growth factor receptor 2-negative, metastatic breast cancer: a roundtable discussion by The Breast Cancer Therapy Expert Group (BCTEG)
}

\author{
Jame Abraham ${ }^{1} \cdot$ Robert Coleman $^{2} \cdot$ Anthony Elias $^{3} \cdot$ Frankie Ann Holmes ${ }^{4} \cdot$ Kevin Kalinsky $^{5} \cdot$ Muaiad Kittaneh $^{6}$. \\ Elyse Lower ${ }^{7} \cdot$ Reshma Mahtani $^{8}$ (D) E. Terry Mamounas ${ }^{9} \cdot$ Mark Pegram $^{10} \cdot$ Charles Vogel $^{8} \cdot$ The Breast Cancer $^{2}$ \\ Therapy Expert Group (BCTEG)
}

Received: 3 April 2018 / Accepted: 6 April 2018 / Published online: 4 May 2018

(c) The Author(s) 2018

\begin{abstract}
Purpose To provide an overview of clinical data supporting the use of cyclin-dependent kinases 4 and 6 (CDK 4/6) inhibitors in the treatment of hormone receptor-positive $(\mathrm{HR}+)$, human epidermal growth factor receptor 2-negative (HER2-), metastatic breast cancer $(\mathrm{mBC})$, from the perspective of the practicing oncologist community.

Methods A recent roundtable discussion was convened by The Breast Cancer Therapy Expert Group (BCTEG) to review existing data on this topic and its impact on their current practice.

Results Level 1 evidence now supports use of a CDK 4/6 inhibitor in combination with endocrine therapy for patients with HR+, HER2-, mBC. Currently, there are no biomarkers that reliably define patients who will, or will not, benefit from the addition of a CDK 4/6 inhibitor to their endocrine therapy. Additional research is needed to identify the optimal sequencing of CDK 4/6 inhibitors in relation to other therapies as well as the optimal duration of therapy; at present, evidence suggests that use in the upfront setting is better than waiting for a later line of therapy, or adding after endocrine therapy has started. Conclusions Thus far, three CDK 4/6 inhibitors-palbociclib, ribociclib, and more recently, abemaciclib-have been approved for use in the setting of HR+, HER2-, mBC. The degrees to which these agents differ in terms of CDK4/6 affinity, side-effect profiles, dosing, degree of central nervous system (CNS) penetration, optimal use in combination with antiestrogen therapy, and across other subsets of breast cancer, remain an active area of investigation.
\end{abstract}

Keywords Breast cancer $\cdot$ Hormone receptor positive $\cdot$ CDK $\cdot$ Metastatic breast cancer $\cdot$ BCTEG $\cdot$ Cyclin-dependent kinase $\cdot$ Endocrine therapy

Electronic supplementary material The online version of this article (https://doi.org/10.1007/s10549-018-4783-1) contains supplementary material, which is available to authorized users.

Reshma Mahtani

rmahtani@miami.edu

Cleveland Clinic, Cleveland, OH, USA

University of Sheffield, Sheffield, England, UK

University of Colorado, Boulder, CO, USA

Texas Oncology, Austin, TX, USA

\section{About the Breast Cancer Therapy Expert Group (BCTEG)}

The BCTEG is a group of expert physicians and clinical researchers who have dedicated their careers to the treatment of patients with breast cancer. The purpose of the group is to

\footnotetext{
Columbia University Medical Center, New York, NY, USA

Loyola University, Chicago, IL, USA

University of Cincinnati, Cincinnati, OH, USA

University of Miami, Deerfield Beach, FL, USA

University of Florida, Gainesville, FL, USA

10 Stanford University, Stanford, CA, USA
} 
meet periodically to discuss important developments related to breast cancer management, with a particular emphasis on new findings and/or areas where guidance from established bodies, such as the National Comprehensive Cancer Network (NCCN) and the American Society for Clinical Oncology (ASCO), may be unresolved, or less well established. The goal is to elicit the group's opinions on a given topic as it relates to their own clinical practices, and more importantly, how this might impact those practicing in the community setting, where breast cancer is only one of many tumor types encountered. Importantly, this article is not intended to replace any existing guidance, or to be construed as an exhaustive review of the topic in question. Rather, its main aim is to present a concise synopsis of the relevant data in this area, and summarize the opinion of the expert group, as gleaned from the meeting discussion.

\section{Meeting objectives and role of funding sources}

In a previous meeting, the group addressed several key issues related to endocrine therapy for hormone receptor-positive $(\mathrm{HR}+)$ early breast cancer (EBC) [1]. A second BCTEG meeting was convened in late August 2017 with the goal of conducting an informal roundtable discussion on the use of cyclin-dependent kinase (CDK) 4/6 inhibitors for HR+ metastatic breast cancer $(\mathrm{mBC})$. An unrestricted educational grant for this activity was provided by Eli Lilly and Company, Pfizer Inc., and Novartis Pharmaceuticals Corporation, with additional support from Total Health Conferencing, a medical education company. The faculty were compensated for their participation, and topics of discussion were selected by the faculty and by Total Health Conferencing. It is recognized that many of the panelists may have relationships with corporate entities, both related and unrelated to the topic in question; content of the discussions, and any expert opinions presented herein, was intended to be based on the panelists' own expert clinical experience and insight, and is understood not to be influenced by any corporate relationship or interest.

\section{Introduction}

Dysregulation of the cell cycle is recognized as a salient feature of cancer cells [2-4]. The cyclin-dependent kinases (CDKs) 4 and 6 play an important regulatory role in the initiation of mitosis in the mammalian cell cycle [2-4]. Under normal conditions, progression through the cell cycle is inhibited by the retinoblastoma gene product ( $\mathrm{pRb}$ ), which represses the E2F transcription factor family and activation of its downstream targets [2, 4]. In response to activating signals such as growth factors or cell adhesion molecules, cyclin D1 is produced, and interacts with CDKs 4 and 6 to form a complex which phosphorylates $\mathrm{pRb}$ and lifts the repression on E2F, allowing it to activate downstream targets, and permitting quiescent cells to irreversibly enter the DNA synthesis (S) phase of the cell cycle [2-4]. There are several findings that have implicated the CDK $4 / 6$ pathway in breast cancers; these include overexpression and/ or amplification of D-type cyclins (e.g. CCNDI) and CDK 4 [2, 3]. Preclinical work in breast cancer cell lines from Finn and coworkers also showed that estrogen receptorpositive $(E R+)$ cell lines were most sensitive to CDK 4/6 inhibition, and evidence for an apparent synergism between CDK 4/6 inhibitors and endocrine therapy (tamoxifen) [2]. Collectively, these findings have led to the development of CDK 4/6 inhibitors as potential therapeutic agents in breast cancer. Whereas first-generation CDK 4/6 inhibitors lacked sufficient specificity, resulting in disappointing clinical efficacy and unacceptable toxicities, second-generation agents are now available that have shown efficacy in breast cancer in both the preclinical and clinical settings; these agents include palbociclib, ribociclib, and abemaciclib [5-14].

\section{CDK 4/6 inhibitors: brief summary of clinical evidence in $\mathrm{mBC}$}

The seminal clinical data supporting the use of CDK $4 / 6$ inhibitors in combination with endocrine therapy for $\mathrm{mBC}$ are summarized in Table 1, and includes the PALOMA-1, -2 , and 3, MONALEESA-2 and -7, and MONARCH-1, -2, and -3 studies for palbociclib, ribociclib, and abemaciclib, respectively. Of note, whereas both palbociclib and ribociclib were approved for use in $\mathrm{mBC}$ at the time of the roundtable discussion, abemaciclib had not yet been approved. Since that time, results from MONARCH-3 have been subsequently been reported, and abemaciclib was approved for use in the mBC setting by the United States Food and Drug Administration (US FDA) based on the MONARCH-1 and 2 trials [15]. As outlined in Table 1, all of these agents have been shown to significantly and robustly improve the endpoint of progression-free survival (PFS) in women with $\mathrm{HR}+\mathrm{mBC}$ when used in combination with selected endocrine therapies. Significant improvement in overall response rate (ORR) and clinical benefit rate (CBR) with the combination therapy (compared to endocrine therapy alone) has also been observed across these studies (Table 1). There are important differences in terms of toxicities with the three agents, which, however, may, at least in part, relate to their differential specificity for the CDKs [3]. Importantly, an overall survival (OS) advantage has not yet been documented in the setting of $\mathrm{mBC}$ (Table 1); thus, the choice of which therapeutic line in which to initiate a CDK 4/6 inhibitor is not mandated. 


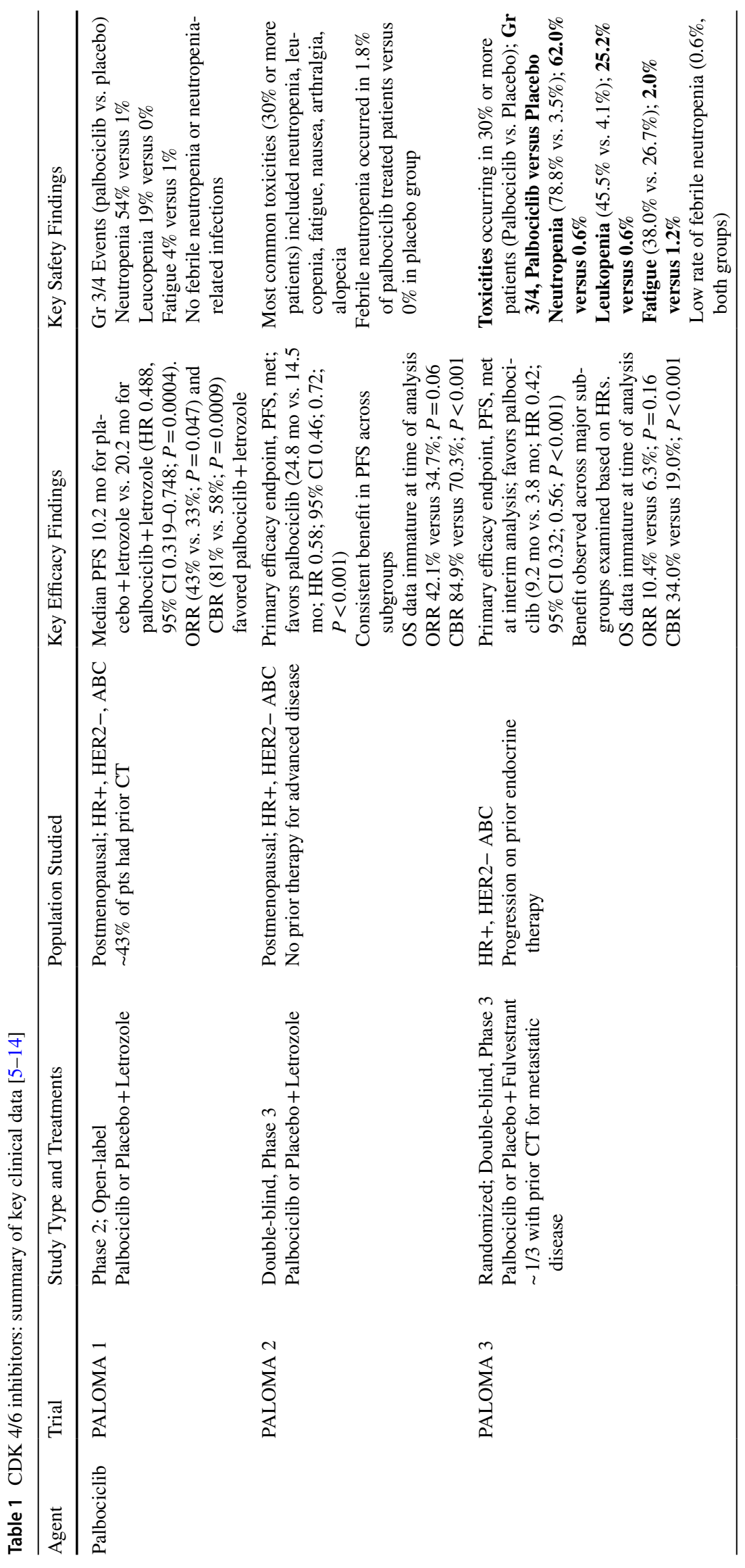




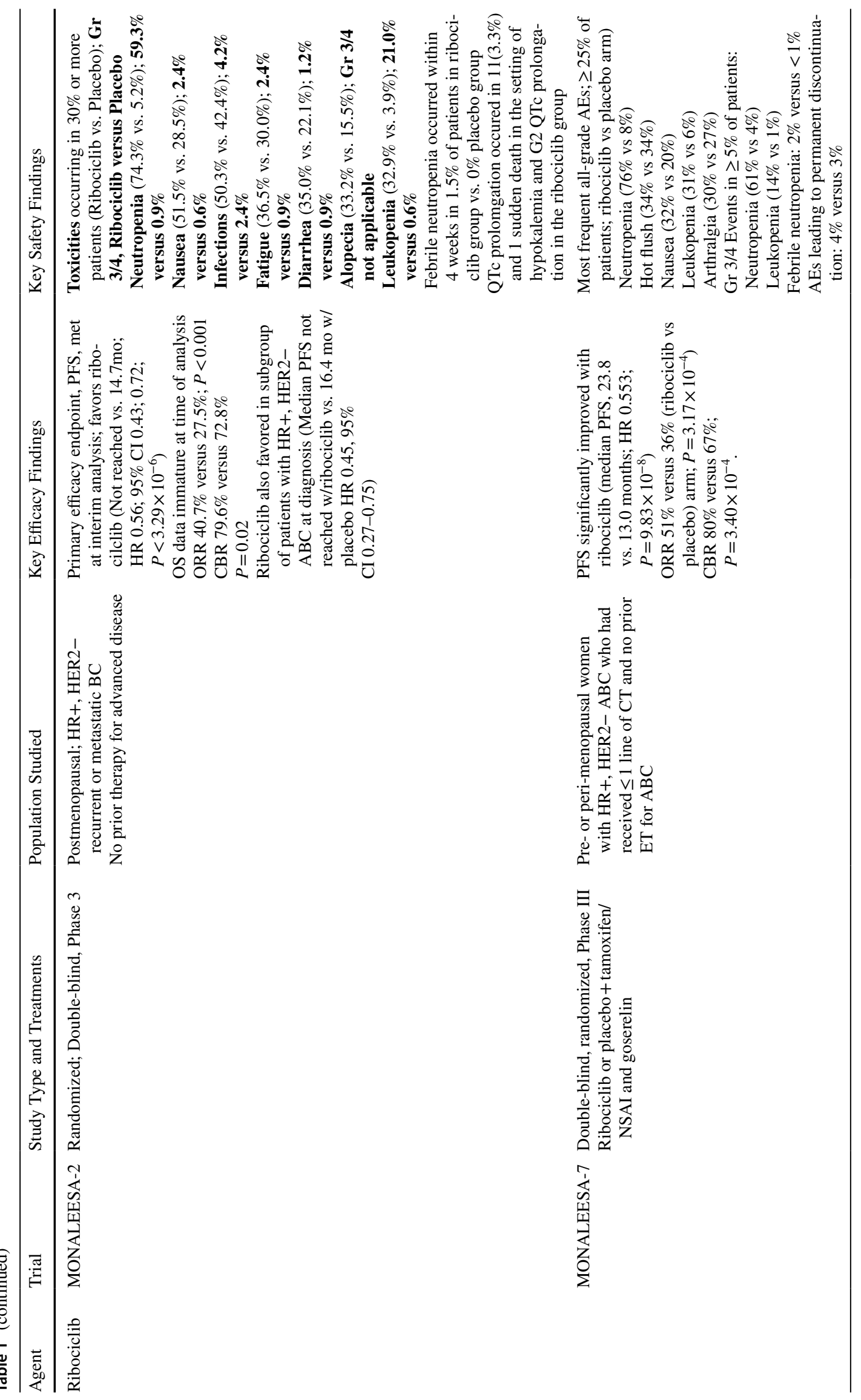




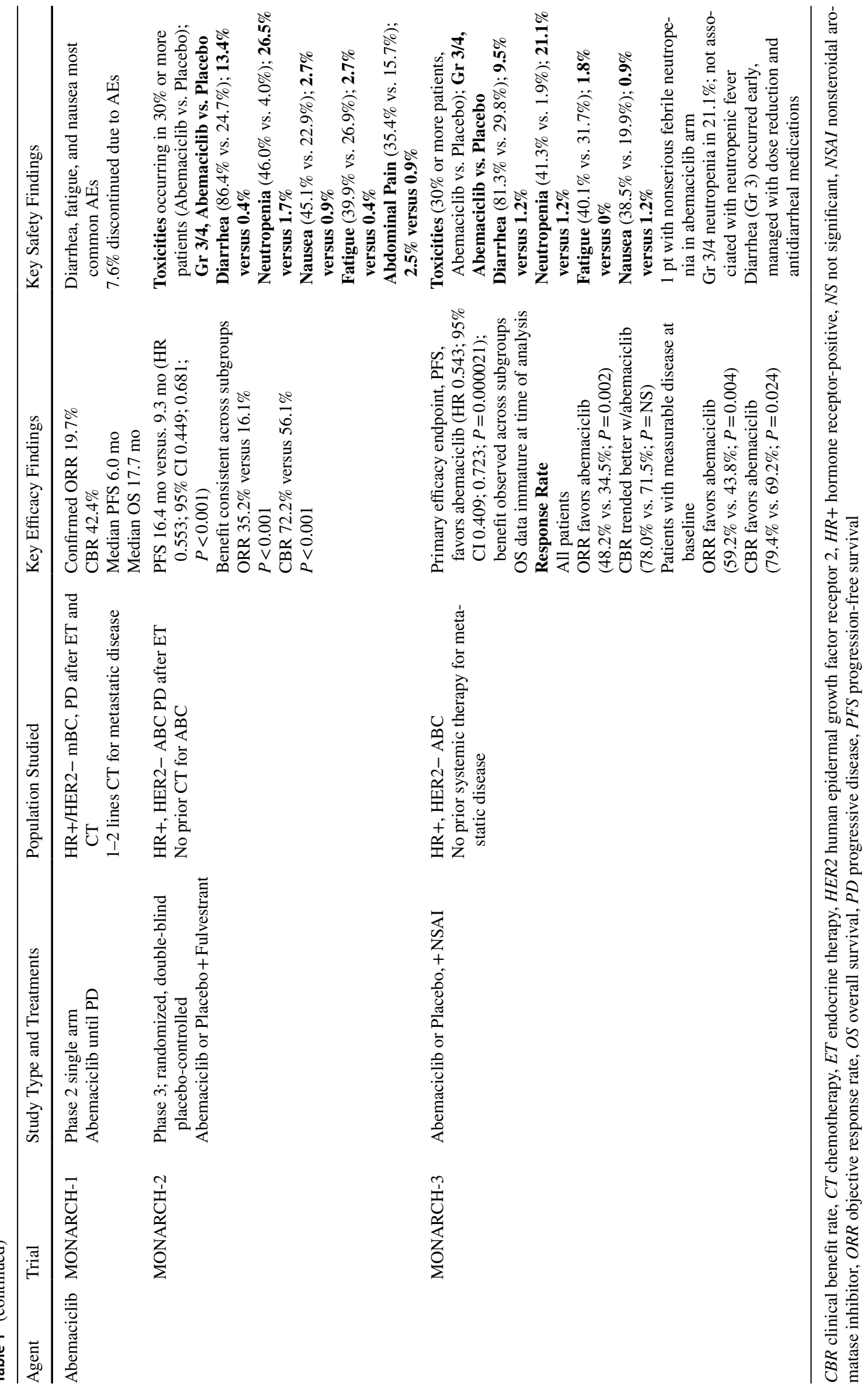




\section{CDK 4/6 treatment: what are the differentiators?}

\section{What are the differentiators, current or future, that drive your decision to treat using the various endocrine options available for the treatment of metastatic breast cancer?}

In terms of PFS reduction (Tables 1), it was agreed by the panel that the three CDK 4/6 inhibitors appear to be largely similar in efficacy, and, as noted above, none has as yet shown a benefit in OS. The initial discussion was focused on the differentiators, current or future, if any, which drive the decision to treat using the various endocrine options available for the treatment of $\mathrm{mBC}$. The group was not entirely uniform with respect to their use of CDK 4/6 inhibitors in the first-line setting for metastatic disease. The principal drivers identified from the discussion included whether the patient was symptomatic or asymptomatic from the metastatic standpoint, whether the metastases were primarily visceral or nonvisceral, menopausal status, and the disease-free interval. Some participants reported (owing to the current lack of OS advantage) being more inclined to use endocrine therapy alone if a patient was asymptomatic with relatively indolent disease and had been off endocrine therapy for a few years, and in those with de novo, bone only metastatic disease. There was general agreement on the early use of a CDK 4/6 inhibitor in younger patients and in those with aggressive, progesterone receptor-negative (PR-) and/or symptomatic disease.

Mutation in ESRl, the gene encoding the estrogen receptor and a suspected marker of endocrine therapy resistance [16-18], was also cited as a potentially meaningful factor to consider, and an active area of research, although among the group, it was not routinely tested for outside of a clinical study, and was not used as a determinant for CDK 4/6 inhibitor use. The group did, however, cite the potential importance of ESRI mutation results, if available, when deciding to use the selective estrogen receptor degrader, fulvestrant or an aromatase inhibitor (AI) in combination with CDK 4/6 therapy [18]. Indeed, results from PALOMA-3 using circulating tumor DNA showed ESR1 mutation in 106 of 395 samples tested (26.8\%), and all of these patients had been previously treated with an $\mathrm{AI}$, whereas no ESRI mutations were found in patients who received tamoxifen only. Also of importance from this study was that median PFS was significantly longer with the combination of palbociclib + fulvestrant as compared to placebo + fulvestrant regardless of patient's ESR1 mutational status [19]. It was noted that new technologies for ESRI mutational testing, such as evaluation of circulating tumor DNA, continue to be developed and, although not currently standard practice, may be routinely incorporated into testing in the near future.

A few participants in the group reported using CDK 4/6 inhibitors as first-line therapy in a majority of patients, citing the robust PFS improvements across all the major studies and within subgroups, in which the doublet was consistently found to be beneficial over the single-agent endocrine therapy. However, the group acknowledged that market research from the major CDK 4/6 developers showed a much lower uptake of these agents than would have been expected, based upon the clinical results; this suggests that clinicians believe they know who will benefit from the drug and who will not, or this could be a manifestation of other variables (e.g., drug cost). In reality, however, there was agreement that, based on Forest plots presented in the major clinical trials, all subgroups of patients with HR+, HER2- mBC benefit, and there is not at present a clinical feature or biomarker for use of these agents, nor is there a clinical outlier whom one could reliably predict would, or would not benefit from the use of a CDK 4/6 inhibitor with endocrine therapy.

With regard to the lack of OS benefit in the face of the robust improvements in PFS, it was suggested that none of the major trials may be sufficiently powered to detect survival benefit, and as such, pooling/meta-analysis of trial data, as well as further follow up, may be needed to adequately evaluate this endpoint. Some participants noted the results of a Southwest Oncology Group (SWOG) study showing improvement in OS with anastrozole and fulvestrant as firstline therapy [20]. Others, however, were more dismissive of the SWOG trial, citing its unplanned subgroup analyses and suggesting the OS benefit was restricted to patients naïve to adjuvant tamoxifen. Another important point raised is that OS benefit has become more difficult to attribute to the first-line therapy, since many possible treatment options are now available to the patient over time; thus determining the impact of a first-line therapy on OS will become progressively more difficult.

\section{Barriers to use of CDK 4/6 inhibitors}

\section{What significant clinical toxicities, financial barriers, and/or common community practice misunderstandings exist regarding the use of CDK 4/6 inhibitors?}

Several barriers to the widespread use of CDK 4/6 inhibitors were identified by the group. Whereas there was agreement that the addition of CDK 4/6 inhibitors to endocrine therapy resulted in overall low additional toxicity over endocrine therapy alone, there was concern over events such as fatigue and alopecia with all of the agents, and diarrhea with 
abemaciclib. In the case of palbociclib, the group expressed some concerns regarding alopecia; some felt incidence of events such as alopecia was similar with ribociclib, although globally the experience with ribociclib in the group was more limited.

The group discussed results presented by Finn and coworkers at 2017 ASCO showing that concerns regarding possible increased incidence or severity of future myelotoxicity upon progression on palbociclib were not substantiated, and indicating that chemotherapy delivery and efficacy post-palbociclib was not compromised [21]. The group felt this was an important point, because if patients were to develop worsened myelotoxicity later in the course of treatment, this would likely compromise the use of subsequent chemotherapies over concerns of further myelosuppression. The group also felt it important to highlight that, despite the frequent occurrence of neutropenia with these agents, the rate of febrile neutropenia was low across the trials, and the group did not report utilizing growth factor or prophylactic antibiotic support with these agents. Patientreported outcome (PRO) and quality of life (QoL) data were also cited from ASCO 2017, which compared ribociclib and placebo (in combination with letrozole) in patients with HR +, HER2- advanced breast cancer (MONALEESA-2 trial). The results of this trial showed (at least in the case of ribociclib) that QoL was not adversely affected, and certain measures (e.g., pain reduction) trended better with the addition of the CDK 4/6 inhibitor [22].

There was discussion surrounding monitoring requirements, and how these impact the choice of CDK4/6 inhibitor.
Electrocardiogram (ECG) monitoring with ribociclib, for example (Table 2), was cited as a nuisance by several members of the group. Regarding the need for complete blood cell (CBC) and other monitoring (Table 2), the panel noted the importance of keeping apprised of any prescribing information updates. Palbociclib, for example, in its most recent update, now recommends $\mathrm{CBC}$ to be monitored prior to the start of therapy and at the beginning of each cycle, as well as on day 15 of the first 2 cycles, and then less frequently (every 3 months) [23]. The update may be beneficial for patients who are not inclined to return monthly for CBC monitoring and for physicians who prefer to follow patients at longer intervals.

Delays in institution of therapy due to the need for dose reductions were also cited as a significant and costly problem. Drug wastage was raised as an important issue in light of rising healthcare costs. Some participants noted that ribociclib dose reduction was easier, owing to its blister packaging. Frequent and appropriate dose reductions were reported as necessary for both hematologic (e.g., neutropenia) and nonhematologic toxicities (e.g., fatigue). Lastly, despite the importance of "having the conversation with the patient" and offering CDK 4/6 inhibitors upfront (in absence of any contraindications), some in the group noted that patients may be inclined to refuse the treatment if the drug costs are prohibitive. Indeed, it was agreed that co-pays of up to $20 \%$ could present a significant financial hardship for many patients, and, given the lack of survival benefit, there was some discussion that PFS benefit alone might not justify the cost. It was also noted by the group that the use of CDK $4 / 6$

Table 2 Currently Approved CDK 4/6 Inhibitors [23, 32, 33]

\begin{tabular}{|c|c|c|c|}
\hline Agent (trade name) & Year approved & Indication & $\begin{array}{l}\text { Most common adverse events and required } \\
\text { monitoring }\end{array}$ \\
\hline Palbociclib (Ibrance ${ }^{\circledR}$ ) & 2015 & $\begin{array}{l}\text { HR+, HER2- Advanced or metastatic breast } \\
\text { cancer, in combination with: } \\
\text { A. An AI as initial endocrine based therapy in } \\
\text { postmenopausal women; or } \\
\text { B. Fulvestrant, in women with disease progres- } \\
\text { sion following endocrine therapy. }\end{array}$ & $\begin{array}{l}\text { (Incidence } \geq 10 \% \text { ) Neutropenia, infections, } \\
\text { leukopenia, fatigue, nausea, stomatitis, anemia, } \\
\text { alopecia, diarrhea, thrombocytopenia, rash, } \\
\text { vomiting, decreased appetite, asthenia, pyrexia } \\
\text { Required Monitoring: CBC }\end{array}$ \\
\hline Ribociclib (Kisqali ${ }^{\circledR}$ ) & 2017 & $\begin{array}{l}\text { Postmenopausal women with HR+, HER2- } \\
\text { Advanced or metastatic breast cancer in } \\
\text { combination with an AI as initial endocrine- } \\
\text { based therapy. }\end{array}$ & $\begin{array}{l}\text { (Incidence }>20 \% \text { ) Neutropenia, nausea, fatigue, } \\
\text { diarrhea, leukopenia, alopecia, vomiting, consti- } \\
\text { pation, headache, back pain } \\
\text { Required Monitoring: QT interval prolongation, } \\
\text { Electrocardiograms (ECGs), Electrolytes, Liver } \\
\text { Function Tests (LFTs), CBC }\end{array}$ \\
\hline Abemaciclib (Verzenio ${ }^{\mathrm{TM}}$ ) & 2017 & $\begin{array}{l}\text { In combination with fulvestrant for } \mathrm{HR}+\text {, } \\
\text { HER2-, Advanced or metastatic breast cancer } \\
\text { with disease progression following endocrine } \\
\text { therapy; or } \\
\text { As monotherapy for HR+, HER2-, Advanced } \\
\text { or metastatic breast cancer with disease } \\
\text { progression following endocrine therapy and } \\
\text { prior chemotherapy in the metastatic setting }\end{array}$ & $\begin{array}{l}\text { (Incidence } \geq 20 \% \text { ) Diarrhea, neutropenia, nausea, } \\
\text { abdominal pain, infections, fatigue, anemia, } \\
\text { leukopenia, decreased appetite, vomiting, head- } \\
\text { ache, thrombocytopenia } \\
\text { Required Monitoring: Liver Function Tests } \\
\text { (LFTs), CBC, Monitor for venous thrombosis } \\
\text { and pulmonary embolism }\end{array}$ \\
\hline
\end{tabular}

$A I$ aromatase inhibitor, $C B C$ complete blood count, $H E R 2$ human epidermal growth factor receptor 2 , $H R$ hormone receptor 
inhibitors may not ultimately be found to be a cost-effective intervention; despite this, however, it was felt that cost alone should not be a deciding factor on whether to use these agents [24-26].

\section{Sequencing Therapies for ER+/HER2- MBC}

\section{How would you approach patients that have progressed on a CDK 4/6 inhibitor?}

With respect to the current status of CDK 4/6 inhibitors in the sequence of treatment, the question was raised about "saving" CDK 4/6 inhibitor use for a later line of therapy. The group cited the higher magnitude of PFS benefit associated with combination therapy upfront, as opposed to using CDK 4/6 inhibitor in combination with fulvestrant at a later time. There was agreement that the first-line therapy a patient receives is generally the one they will continue for the longest period of time, and that, in accordance with the general principles of oncology, the most effective, and the least toxic therapies should be used first. The group then discussed the management of patients who progress on AI monotherapy after more than 1 year of disease control. Possible considerations included considering ESRI mutational status to determine if a switch to fulvestrant would be appropriate. Fulvestrant therapy was also thought to be beneficial in this scenario as it assures compliance due to its mode of administration. They acknowledged results from the To Reverse ENDocrine resistance (TREND) trial, which demonstrated that highly selected patients may benefit from adding palbociclib to long-term AI therapy after progression (with no change in endocrine therapy), with the caveat that this was a small study $(N=115)$ and the approach should not be used routinely in the absence of larger confirmatory data [27]. This trial also showed activity of single-agent palbociclib in this setting (CBR, 60\%), and in this regard, it should also be noted that all three agents have been shown to have at least some activity as a monotherapy, although at present, only abemaciclib is approved as a single-agent treatment (Table 2) [2-4, 10, 27-29]

The group was generally in agreement on the upfront use of an AI with a CDK 4/6 inhibitor, followed by (upon progression) fulvestrant or exemestane with a mammalian target-of-rapamycin (mTOR) inhibitor (e.g. everolimus). The incorporation of everolimus was felt to be associated with additional toxicity, albeit manageable toxicity with the incorporation of additional measures (e.g., steroid-based mouthwash). The data supporting the combination of fulvestrant and everolimus were cited as the PrECOG 0102 data presented at San Antonio Breast Cancer Symposium (SABCS) 2016 [30]. Notably, patients in this study had not been treated with prior CDK 4/6 inhibitors, and another important unresolved issue noted by the group (and not addressed in PrECOG 0102) was whether CDK 4/6 inhibitor therapy should be continued upon progression.

There was agreement that data on switching between CDK 4/6 inhibitors are not yet available, and while the label for abemaciclib does not exclude patients who previously received a CDK4/6 inhibitor, the Monarch-1 study did not allow prior CDK4/6 inhibitors. Efficacy data for abemaciclib monotherapy following progression on a CDK $4 / 6$ inhibitor are therefore not yet available. The potential use of CDK 4/6 inhibitors in combination with tamoxifen in pre- and perimenopausal women was also discussed, and in this regard, the panel now recognizes recently reported results from MONALEESA-7 (SABCS 2017) which confirm a benefit of using ribociclib in combination with tamoxifen (and goserelin) in this patient population (Table 1) [14]. At present, there are no data on using tamoxifen (without ovarian suppression) and a CDK 4/6 inhibitor.

\section{Biomarkers for CDK 4/6 inhibitors}

\section{What biomarkers, if any, have been shown to predict benefit, or lack thereof, when using CDK4/6 inhibitors (e.g., ESR1, CND1 amplification, p16 loss, or RB1 expression)?}

Regarding the use of biomarkers to predict responses in patients treated with CDK 4/6 inhibitors, the panel noted the results from PALOMA-1, in which markers such as cyclin D1 amplification and p16 loss did not reliably predict response to palbociclib [5, 31]. They agreed clinicians should not use biomarkers to make treatment decisions, and the only proven biomarker of response per se is the estrogen receptor itself. The group also recognized the need for phosphorylated $\mathrm{pRb}$ in order for the CDK $4 / 6$ inhibitors to work, and some of the group said they would be reluctant to start on a CDK 4/6 inhibitor, if it was known the tumor had loss of $\mathrm{pRb}$. This is perhaps most relevant for a patient that had progressed on singleagent AI and for whom such information could be potentially available, whereas for most patients this would not be known in the up front setting. The group thought that markers of response early in the course of single-agent therapy are urgently needed, and cited efforts underway to find markers such as mutational load in serum during therapy. At present, however, it was agreed that such markers are not available. 


\section{Summary and key points}

Overall, the group viewed the addition of CDK 4/6 inhibitors as a valuable therapeutic option available to women with $\mathrm{HR}+$, HER2-, mBC, with the caveat that additional research is needed to gain clarification on several important issues such as treatment biomarkers, optimal duration of therapy, continuation of CDK4/6 inhibitors, and/or a switch to a different drug in the same class following disease progression. In light of this BCTEG roundtable discussion, the following expert opinion statements can be made:

- Level 1 evidence now supports use of a CDK 4/6 inhibitor in combination with endocrine therapy for patients with HR+, HER2-, mBC as first-line treatment.

- Currently, there are no biomarkers that reliably define patients who will or will not benefit from the addition of a CDK 4/6 inhibitor to endocrine therapy; ESR1 mutational status should not restrict use of a CDK 4/6 inhibitor.

- Financial considerations may limit the use of these agents, and treatment-related toxicities such as diarrhea and fatigue may be dose limiting; although neutropenia is a frequent occurrence with these agents, the rate of febrile neutropenia is low.

- Additional research is needed to identify the optimal sequencing of CDK 4/6 inhibitors in relation to other therapies as well as optimal duration of therapy; at present, use in the upfront setting is better than waiting for a later line of therapy or adding after endocrine therapy has started.

Finally, the group agreed that CDK 4/6 inhibitors are an important new class of drugs in the management of patients with inoperable breast cancer. In addition, it was recognized that at present, breast cancer is leading the field in clinical experience with CDK 4/6 inhibitors, and research currently underway in breast cancer (e.g., optimal duration of therapy, use in combination with other treatments) will be of value to many other cancer types such as lung cancer, glioblastoma, and melanoma. It was noted, for example, that, although their use in combination with endocrine therapy is now well established, due to their antiproliferative activity, CDK 4/6 inhibitors might not be optimally combined with other emergent therapies, such as immunotherapy (which requires $\mathrm{T}$ cell proliferation), and might in fact act antagonistically with some types of chemotherapy. These and other combinations await further evaluation in clinical trials.

Funding An unrestricted educational Grant for this activity was provided by Eli Lilly and Company, Pfizer Inc., and Novartis Pharmaceuticals Corporation, with additional support from Total Health Conferencing, a medical education company. The faculty were compensated for their participation. Independent medical writing support was provided by SciavoTECH Research and Consultancy Services, Inc. and funded by Total Health Conferencing.

Open Access This article is distributed under the terms of the Creative Commons Attribution 4.0 International License (http://creativeco mmons.org/licenses/by/4.0/), which permits unrestricted use, distribution, and reproduction in any medium, provided you give appropriate credit to the original author(s) and the source, provide a link to the Creative Commons license, and indicate if changes were made.

\section{References}

1. Abraham J, Caldera H, Coleman R et al Breast Cancer Therapy Expert Group (BCTEG) (2018) Endocrine therapy and related issues in hormone receptor-positive early breast cancer: a roundtable discussion by the breast cancer therapy expert group (BCTEG). Breast Cancer Res Treat. https://doi.org/10.1007/s1054 9-018-4662-4669

2. Finn RS, Aleshin A, Slamon DJ (2016) Targeting the cyclindependent kinases (CDK) 4/6 in estrogen receptor-positive breast cancers. Breast Cancer Res 18(1):17. https://doi.org/10.1186/ s13058-015-0661-5

3. Barroso-Sousa R, Shapiro GI, Tolaney SM (2016) Clinical development of the CDK4/6 inhibitors ribociclib and abemaciclib in breast cancer. Breast Care (Basel). 11(3):167-173. https://doi. org/10.1159/000447284

4. Xu H, Yu S, Liu Q, Yuan X, Mani S, Pestell RG, Wu K (2017) Recent advances of highly selective CDK4/6 inhibitors in breast cancer. J Hematol Oncol 10(1):97. https://doi.org/10.1186/s1304 5-017-0467-2

5. Finn RS, Crown JP, Lang I et al (2015) The cyclin-dependent kinase $4 / 6$ inhibitor palbociclib in combination with letrozole versus letrozole alone as first-line treatment of oestrogen receptorpositive, HER2-negative, advanced breast cancer (PALOMA-1/ TRIO-18): a randomised phase 2 study. Lancet Oncol 16(1):2535. https://doi.org/10.1016/S1470-2045(14)71159-3

6. Turner NC, Ro J, André F, Loi S, Verma S, Iwata H, Harbeck N, Loibl S, Huang Bartlett C, Zhang K, Giorgetti C, Randolph S, Koehler M (2015) Cristofanilli M; PALOMA3 Study Group. Palbociclib in hormone-receptor-positive advanced breast cancer. N Engl J Med 373(3):209-219. https://doi.org/10.1056/NEJMo a1505270

7. Cristofanilli M, Turner NC, Bondarenko I et al (2016) Fulvestrant plus palbociclib versus fulvestrant plus placebo for treatment of hormone-receptor-positive, HER2-negative metastatic breast cancer that progressed on previous endocrine therapy (PALOMA-3): final analysis of the multicentre, double-blind, phase 3 randomised controlled trial. Lancet Oncol 17(4):425-439. https ://doi.org/10.1016/S1470-2045(15)00613-0

8. Finn RS, Martin M, Rugo HS et al (2016) Palbociclib and letrozole in advanced breast cancer. N Engl J Med 375(20):1925-1936

9. Hortobagyi GN, Stemmer SM, Burris HA et al (2016) Ribociclib as first-line therapy for hr-positive, advanced breast cancer. N Engl J Med 375(18):1738-1748

10. Dickler MN, Tolaney SM, Rugo HS et al (2017) MONARCH 1, a phase II study of abemaciclib, a CDK4 and CDK6 inhibitor, as a single agent, in patients with refractory $\mathrm{HR}(+) / \mathrm{HER} 2(-)$ metastatic breast cancer. Clin Cancer Res 23(17):5218-5224. https:// doi.org/10.1158/1078-0432.CCR-17-0754

11. Di Leo A, Toi M, Campone M, et al. (2017) MONARCH 3: Abemaciclib as initial therapy for patients with $\mathrm{HR}+$ / 
HER2- advanced breast cancer, Ann Oncol, 2017. https://doi. org/10.1093/annonc/mdx365 [Abstract 236O]

12. O'Shaughnessy J, Petrakova K, Sonke GS et al (2017) Ribociclib plus letrozole versus letrozole alone in patients with de novo HR +, HER2- advanced breast cancer in the randomized MONALEESA-2 trial. Breast Cancer Res Treat. https://doi. org/10.1007/s10549-017-4518-8

13. Sledge GW, Toi M, Neven P et al (2017) MONARCH 2: abemaciclib in combination with fulvestrant in women with $\mathrm{HR}+/$ HER2-advanced breast cancer who Had progressed while receiving endocrine therapy. J Clin Oncol 35(25):2875-2884

14. Tripathy D, Sohn J, Im S-A, et al. (2017) First-line ribociclib vs placebo with goserelin and tamoxifen or a non-steroidal aromatase inhibitor in premenopausal women with hormone receptor-positive, HER2-negative advanced breast cancer: Results from the randomized phase III MONALEESA-7 trial. San Antonio Breast Cancer Symposium 2017. Abstract GS2-05

15. ASCO Post: FDA Approves New CDK4/6 Inhibitor for Certain Advanced or Metastatic Breast Cancers. http://www.ascopost. com/News/58090?utm_medium=Email\&utm_source=ExactTarge t\&utm_campaign=\&utm_term=7064487 Accessed 24 Nov 2017

16. Jeselsohn R, Buchwalter G, De Angelis C, Brown M, Schiff R (2015) ESR1 mutations-a mechanism for acquired endocrine resistance in breast cancer. Nat Rev Clin Oncol. 12(10):573-583. https://doi.org/10.1038/nrclinonc.2015.117

17. Wardell SE, Ellis MJ, Alley HM et al (2015) Efficacy of SERD/SERM Hybrid-CDK4/6 inhibitor combinations in models of endocrine therapy-resistant breast cancer. Clin Cancer Res 21(22):5121-5130. https://doi.org/10.1158/1078-0432. CCR-15-0360

18. Fribbens C, O'Leary B, Kilburn L et al (2016) Plasma ESR1 mutations and the treatment of estrogen receptor-positive advanced breast cancer. J Clin Oncol 34(25):2961-2968. https:// doi.org/10.1200/JCO.2016.67.3061

19. Turner NC, Jiang Y, O’Leary B, et al. (2016) Efficacy of palbociclib plus fulvestrant $(\mathrm{P}+\mathrm{F})$ in patients (pts) with metastatic breast cancer (MBC) and ESR1 mutations (mus) in circulating tumor DNA (ctDNA). J Clin Oncol 34(15_suppl):512-512

20. Mehta RS, Barlow WE, Albain KS et al (2012) Combination anastrozole and fulvestrant in metastatic breast cancer. N Engl J Med 367(5):435-444. https://doi.org/10.1056/NEJMoa1201622

21. Finn RS, Crown J, Lang I, et al. (2017) Overall survival results from the randomized phase II study of palbociclib $(\mathrm{P})$ in combination with letrozole $(\mathrm{L})$ vs letrozole alone for frontline treatment of ER+/HER2- advanced breast cancer (PALOMA-1; TRIO-18). J Clin Oncol 35:(15_suppl):1001-1001

22. Verma S, O'Shaughnessy J, Burris HA et al (2017) Health-related quality of life (HRQoL) of postmenopausal women with hormone receptor-positive $(\mathrm{HR}+)$, human epidermal growth factor receptor 2-negative (HER2-) advanced breast cancer (ABC) treated with ribociclib + letrozole. J Clin Oncol 35:1020-1020. https://doi. org/10.1200/jco.2017.35.15_suppl.1020

23. IBRANCE ${ }^{\circledR}$ (palbociclib) capsules. Prescribing information. Revised 4/2017. Pfizer, Inc. New York, NY

24. Matter-Walstra K, Ruhstaller T, Klingbiel D, Schwenkglenks M, Dedes KJ (2016) Palbociclib as a first-line treatment in oestrogen receptor-positive, HER2-negative, advanced breast cancer not cost-effective with current pricing: a health economic analysis of the Swiss Group for Clinical Cancer Research (SAKK). Breast Cancer Res Treat 158(1):51-57. https://doi.org/10.1007/s1054 9-016-3822-z

25. Mamiya H, Tahara RK, Tolaney SM, Choudhry NK, Najafzadeh M (2017) Cost-effectiveness of palbociclib in hormone receptorpositive advanced breast cancer. Ann Oncol 28(8):1825-1831. https://doi.org/10.1093/annonc/mdx201

26. Raphael J, Helou J, Pritchard KI, Naimark DM (2017) Palbociclib in hormone receptor -positive advanced breast cancer: a cost-utility analysis. Eur J Cancer 85:146-154. https://doi.org/10.1016/j. ejca.2017.08.018

27. Malorni L, Curigliano G, Minisini AM, et al. (2017) A phase II trial of the CDK4/6 inhibitor palbociclib $(\mathrm{P})$ as single agent or in combination with the same endocrine therapy (ET) received prior to disease progression, in patients (pts) with hormone receptor positive $(\mathrm{HR}+)$ HER2 negative (HER2-) metastatic breast cancer (mBC) (TREnd trial). J Clin Oncol 35 (suppl; abstr 1002)

28. Infante JR, Shapiro G, Witteveen P, Gerecitano JF, Ribrag V, Chugh R et al (2014) A phase I study of the singleagent CDK4/6 inhibitor LEE011 in pts with advanced solid tumors and lymphomas. J Clin Oncol 32(15_suppl):2528-2528

29. DeMichele A, Clark AS, Tan KS et al (2015) CDK 4/6 inhibitor palbociclib (PD0332991) in Rb+ advanced breast cancer: phase II activity, safety, and predictive biomarker assessment. Clin Cancer Res 21(5):995-1001

30. Kornblum NS, Manola J, Klein P et al (2017) PrECOG 0102: A randomized, double-blind, phase II trial of fulvestrant plus everolimus or placebo in post-menopausal women with hormone receptor (HR)-positive, HER2-negative metastatic breast cancer (MBC) resistant to aromatase inhibitor (AI) therapy. Cancer Res. https://doi.org/10.1158/1538-7445.sabcs16-s1-02

31. Cognetti F, Malaguti P, Alesini D (2016) Palbociclib: efficacious but predictive biomarkers still needed. Lancet Oncol 17(4):402403. https://doi.org/10.1016/S1470-2045(16)00144-3

32. KISQALI ${ }^{\circledR}$ (ribociclib) tablets. Prescribing information. Novartis Pharmaceuticals Corporation. East Hanover, New Jersey

33. VERZENIO ${ }^{\mathrm{TM}}$ (abemaciclib) tablets. Prescribing information. Eli Lilly and Company, Indianapolis 